\title{
Jóvenes universitarios mexicanos y la formación de la ciudadanía a través de las redes sociales
}

\author{
Autor: \\ Alberto Farías Ochoa \\ http://orcid.org/0000-0002-8630-6988 \\ Universidad Michoacana de San Nicolás de Hidalgo \\ Mexico \\ Facultad de Letras, \\ Profesor Investigador Asociado
}

\section{RESUMEN}

El presente artículo es un reporte de investigación culminada que centra su mirada en las interacciones establecidas entre jóvenes universitarios de Michoacán (México), y sus capacidades de socialización, negociación y educación, entre pares, a través del entorno virtual Facebook. El objetivo fue indagar si dichos jóvenes son capaces de propiciar actos educativos orientados a la formación de ciudadanía en sus dimensiones civil, social y política entre sus iguales a través de la tecnología. El contexto social durante el proceso de la investigación estaba marcado por una alta actividad política, a través de comicios electorales, y por condiciones de constante violencia en el entorno. La metodología empleada para el seguimiento de las interacciones fue de carácter cualitativo, y con base en la netnografía, incorporando también herramientas del análisis del discurso para el estudio de

\section{ABSTRACT}

This article is a report of a culminated research which focused on the interactions between university students of Michoacan (Mexico), and, on their socialization, negotiation and education skills between peers, through the virtual environment on Facebook. The purpose was to investigate whether these young people are able to promote educational events related to citizenship construction on the civil, social and political dimensions among peers through technology. The social context during the investigation process was marked by a high political activity, through elections, and conditions of constant violence in the environment. The methodology used for monitoring the interactions was qualitative, and it was based on the netnography method, also, some tools of speech analysis were incorporated for studying the recorded conversations, which were reconstructed through the co- 
las conversaciones registradas, las cuales fueron reconstruidas a través de la recopilación, y sistematización, de los mensajes que se intercambiaban en un grupo específico de Facebook. Entre otros resultados se obtuvo evidencia de que los jóvenes en observación sí socializan problemáticas relacionadas con la construcción de ciudadanía, principalmente aquellas que están vinculadas con sus preocupaciones en el entorno, sin embargo, son pocas las interacciones que propician un acto educativo entre pares que pudiese propiciar transformación de los significados.

PALABRAS CLAVE: Formación de ciudadanía; interacción mediática; jóvenes universitarios; tecnologías

\section{INTRODUCCIÓN}

En este trabajo se exponen los resultados de investigación sobre las formas de comunicación y de interacción de los jóvenes universitarios mexicanos en las redes sociales. Fija la mirada en las acciones comunicativas y relacionales, en razón de que el intercambio de mensajes en este tipo de plataformas ha transformando las prácticas de socialización, de gestión, de denuncia y de participación ciudadana de los jóvenes a nivel global.

Se revisa la situación de conectividad, comunicación e interacción de los universitarios del Estado de Michoacán, México, y específicamente de los jóvenes de la Universidad Michoacana San Nicolás de Hidalgo (UMSNH), entre los que según la investigación de Cabrera (2014: 77) el 100\% de los consultados manifiesta tener acceso a internet y telefonía celular. Aunado a lo anterior, se refuerza la idea con los datos de Cruz Yépez (2013) quien reporta que los miembros de esta población aceptan participar en las redes sociales activamente, utilizando principalmente Facebook, atendiendo contenidos en el siguiente orden: distracción, informativos, académicos y, recientemente, de acción política. llection, and systematization, of the messages that had been exchanged in a specific group of Facebook. Among other results, there is evidence that young people under observation usually socializes, trough the virtual environment of Facebook, several problems related to the construction of citizenship, especially those problems that are related to their concerns of the society. Despite of the last, there were just a few interactions that promoted an educational act between peers which could promote transformation of meanings.

KEY WORDS: Citizenship construction; media interactions; technology; university young people

En el estudio sobre cultura juvenil contemporánea de Padilla de la Torre (2013: 127), se enfatiza el hecho de la acción política y se registra que los jóvenes utilizan distintos recursos de conectividad como las redes sociales, o los blogs, para crear relaciones con sus pares y construir comunidades afines a sus intereses y preocupaciones. Al respecto, Padilla (2013) propone que este fenómeno se debe analizar con relación a tres espacios:

1. En la política institucional formal, 2. en la subpolítica, que se define como la participación en organizaciones civiles y movimientos sociales, y 3. en el nivel del subactivismo, que significa indagar en la esfera de lo cotidiano y privado la gestación y emergencia de los sentidos políticos (Padilla, 2013: 131).

En relación a la potencialidad organizativa de las redes sociales resulta útil hacer alusión a las prácticas de comunicación, organización y gestión de los jóvenes de diversas partes del mundo, como los de Egipto, Reino Unido, Grecia, Islandia, España, Chile, entre otros, quienes en años recientes han utilizado la tecnología como medio de comunicación no controlado por instituciones 
del statu quo para compartir sus opiniones, exponer sus demandas y plantear formas de organización y participación con el propósito de resolverlas, así como lo apunta Castells (2012: 37-64).

El vigente interés de los jóvenes universitarios de México por el uso de las redes sociales para la denuncia, la organización y la participación es asimilable desde distintas miradas. Por ejemplo, durante los últimos años este país ha vivido en un clima de creciente violencia debido al establecimiento del narcotráfico en parte del territorio, sumado a los efectos sociales de la llamada crisis de las instituciones, que de acuerdo con la postura de Robinson (2007: 51), ha afectado de manera negativa las formas de la vida social.

Al inicio de esta investigación (2011), los jóvenes universitarios del Estado de Michoacán vivían una coyuntura marcada por los comicios electorales en un entorno de violencia por los enfrentamientos crecientes de las fuerzas del orden con el narcotráfico; sumado a las difíciles condiciones económicas y la falta de oportunidades de trabajo que obstaculizan su desarrollo profesional. En esta coyuntura de malestar social, los jóvenes universitarios de Michoacán, al igual que sus congéneres a nivel nacional y global, utilizan las redes sociales para organizarse, exponer sus preocupaciones, sus necesidades y proponer soluciones.

Dada la problemática interesó a esta investigación indagar sobre las formas de comunicación e interacción que se dan en un grupo específico de la red social: el grupo de Ciudadanos por Michoacán en Facebook. Este grupo estaba integrado mayoritariamente por miembros de la Universidad Michoacana de San Nicolás de Hidalgo, y en su autodenominación como grupo manifiestan el propósito de generar un espacio de discusión en torno a los problemas del Estado de Michoacán.

Con estas características y propósitos del grupo, la investigación buscó observar si el mismo se conformaba como una comunidad de práctica y aprendizaje, en cuyas dinámicas de intercambio sobre temáticas relacionadas con los problemas del Estado de Michoacán, se daban actos educativos de tipo informal entre sus participantes con relación a la formación de ciudadanía, comprendiéndola como el proceso de conocimiento y puesta en práctica de sus derechos y obligaciones, que demuestre:

La capacidad del individuo de reconocerse como parte de una comunidad, en donde su participación es trascendente para el logro de bienes comunes con la finalidad de mejora de los escenarios públicos. Asumiendo la práctica de derechos y obligaciones como garantías de la democracia, y propiciando un sentido de equidad, justicia y ética en la cotidianidad de sus acciones (Fernández, 2010: 47).

Bajo esta mirada, se analizaron aquellas interacciones identificadas como actos educativos informales, en los cuales la educación informal se comprende como el proceso "...que dura toda la vida y en el que las personas adquieren y acumulan conocimientos, habilidades, actitudes y modos de discernimiento mediante las experiencias diarias y en relación con su medio ambiente" (Coombs y Ahmed en Sarramona, Vázquez, y Colom 1998: 12).

En síntesis, el propósito de la investigación fue indagar si los jóvenes de la comunidad virtual Ciudadanos por Michoacán, mediante el diálogo constante y la problematización de su mundo en un grupo creado ex profeso para ello en Facebook, propiciaban actos educativos informales orientados a la formación de ciudadanía entre pares. Para dichos propósitos se partió del siguiente objetivo general de investigación:

Analizar en el grupo Ciudadanos por Michoacán en Facebook, las interacciones de los universitarios de Michoacán que producen actos o procesos de educación informal entre pares, relacionados con las temáticas 
de la formación de la ciudadanía, esto es, adquirir conocimientos sobre los derechos y obligaciones de los miembros de una comunidad, sea ésta local o nacional; y la participación, que no es sino la práctica de la condición ciudadana, en las ámbitos civil, social y político.

En este orden de ideas, relativo a la construcción de una noción de ciudadanía, vinculada a la normativa constitucional y a los escenarios de convivencia en los cuales se incluyen aspectos éticos, se tomó como referencia la postura de la ONU la cual determina cinco aspectos relevantes para la noción de ciudadanía como las dimensiones civil, política, social, económica, y cultural; todas expresadas en las Constituciones Nacionales y en los Pactos Internacionales de Derechos Humanos, de esta manera, para fines del análisis en este trabajo se recuperan tres, que son las dimensiones civil, política y social.

El análisis de las interacciones incluidas en esta investigación se ubicó en la faceta de una comunidad de práctica orientada al aprendizaje, en donde se parte de reconocer a la comunidad de práctica como una modalidad histórica de aprendizaje, mediante la cual el ser humano apropia conocimientos dentro de su entorno.

Se parte del concepto de práctica como la acción que va más allá de hacer algo en sí mismo, es el hacer algo dentro de un contexto histórico y social otorgándole estructura y significado a eso que se hace, lo que de acuerdo con Wenger es siempre una práctica social (Wenger, 2001: 71).

\section{METODOLOGÍA}

En el entendido de que el presente trabajo se trata de una investigación inductiva, de tipo exploratoria y con propósitos descriptivos, las estrategias y técnicas fueron diseñadas en función del objeto de estudio (las interacciones entre los jóvenes universitarios) y las condiciones particulares que éste presenta.
Para la recopilación de las interacciones se seleccionaron dos momentos de registro, los cuales corresponden al seguimiento de las aportaciones en el grupo Ciudadanos por Michoacán durante dos periodos de coyuntura electoral, periodos en los que se generó una abundante participación por parte de los usuarios con temáticas relacionadas a la acepción de ciudadanía.

Aunado a lo anterior, la estrategia metodológica que se persiguió en el presente trabajo se dio bajo un enfoque cualitativo por lo que el interés se centró en las condiciones en las que se desarrollaban los diálogos entre los jóvenes universitarios, la descripción de las conversaciones, la caracterización de los participantes, los tonos y formas en los relatos, la importancia asignada a los temas relacionados con la ciudadanía y las referencias de los roles entre los participantes.

Se estructuraron dos etapas metodológicas, en una primera etapa de registro se diseñó una técnica con base en los principios de la netnografía, en la cual se prestó atención a la relación de los textos generados en el grupo con el contexto de los participantes, al intercambio de los mensajes que lograban transformarse en interacciones, y a los actos comunicativos en general que transcurrieron en el grupo con la finalidad de identificar aquellos que guardaban relación con las dimensiones de ciudadanía consideradas.

Mediante el primer instrumento diseñado se registraron todas las interacciones que se presentaron en el grupo Ciudadanos por Michoacán, con el propósito de identificar la forma en la que los jóvenes participantes se comunican a través del grupo, y los temas que someten a discusión.

En la segunda etapa, se incorporó como técnica el análisis discursivo con orientación a los aspectos de la netnografía. Durante esta etapa el interés se centró en aquellas conversaciones que sí guardaban relación con las dimensiones de ciudadanía estudiadas y en las que participan los jóvenes nicolaitas 
usuarios del grupo, en los roles que juegan dichos jóvenes dentro de las conversaciones, y en la identificación de las acciones del proceso de aprendizaje posible.

Durante la primera etapa se registraron un total de 220 aportaciones originales de diversos temas publicadas en el muro del grupo Ciudadanos por Michoacán. En lo correspondiente a la segunda etapa se registraron solamente 70 aportaciones originales, esto debido a que se tomaron en cuenta solamente las relacionadas con las dimensiones de ciudadanía consideradas, pero se registraron también un total de 133 comentarios-respuesta lo que permitió la construcción de las conversaciones entre los usuarios.

De acuerdo con los objetivos de esta investigación, se comprende la netnografía, desde la noción de Trupo (2011: 3), como un método de análisis de los contenidos en entornos virtuales en internet. Dicho método de análisis permite la identificación, seguimiento y, en su caso, la interpretación de diferentes líneas discursivas que emplean los usuarios de internet respecto a ciertos temas de su interés.

\subsection{SUJETOS DE ESTUDIO}

Los sujetos de estudio en esta investigación son los jóvenes universitarios integrantes del grupo Ciudadanos por Michoacán. Se seleccionaron estos jóvenes debido a que presentan las siguientes características:

a) son los jóvenes de 17 a 29 años de edad quienes dedican más tiempo al sitio Facebook;

b) manifiestan mayor contacto con artefactos tecnológicos;

c)dan muestra de la incorporación de Facebook para actividades de su vida cotidiana, como las de organización de actividades e intercambio de recursos;

d) se involucran en iniciativas promovidas por otros jóvenes de su nación o de otras partes del mundo;

e) generan canales alternativos de expresión con base en sus necesidades; f) se atreven a generar denuncia y de- mandar mejores condiciones a través de estos entornos virtuales; y

g) son integrantes de un grupo orientado al debate de asuntos de ciudadanía (Grupo de Facebook Ciudadanos por Michoacán).

Los universitarios nicolaitas(1), y otros de la entidad federativa, generan actividades de socialización y coordinación entre pares para diversos fines en los entornos virtuales, por ejemplo, han manifestado sus preocupaciones a través de los espacios considerados como públicos, como algunas de las redes sociales, en las que intercambian opiniones y se retroalimentan en función de sus demandas y necesidades.

Se pueden observar algunos casos como el de Indignaos Universitarios de Michoacán en Facebook, cuya principal preocupación es el tema de la violencia y la presencia de las fuerzas armadas en el estado. Aunado a lo anterior, cabe señalar que los jóvenes universitarios de Michoacán destacan por su alto nivel de actividad política; es decir, de participación de manera institucional, y fuera de la institución, en los asuntos públicos de la sociedad incidiendo en la dinámica cotidiana en la toma de decisiones.

El seguimiento en la red social de las distintas organizaciones en las que participan los jóvenes nicolaitas generó una complejidad particular, lo anterior debido a que en cada momento de participación se multiplicaban las posibilidades de análisis, es por ello que en esta investigación se decidió trabajar con un formato específico que permitió concentrar las participaciones de dichos jóvenes encaminadas en objetivos similares. La posibilidad de seguimiento a los jóvenes usuarios del grupo de Facebook otorgó las siguientes ventajas para la sistematización de la información:

a) Publicación de los mensajes en un solo muro a disposición de todos los miembros del grupo.

b) Identificación de los participantes como usuarios individuales de Facebook. 
c) Integración de distintos formatos de contenido; fotos, video, audio, texto e hipervínculos.

d) Generación de documentos y archivo histórico de las aportaciones y discusiones, a disposición de los usuarios. e) Promoción de eventos y convocatorias con base en un calendario a disposición de los usuarios participantes.

Debido a la posibilidad de participación abierta y la cantidad de diversas temáticas incorporadas a las discusiones, la estrategia para recopilar la información se diseñó desde la observación de los participantes en el mismo grupo, indagando entre otros aspectos cualitativos, la participación de los integrantes desempeñando diferentes roles o papeles, las diferencias del entorno virtual en contraste con lo que sucede en el mundo presencial en el mismo contexto histórico, las diferencias en el nivel de interés que muestran los participantes con las diferentes temáticas.

Con la finalidad de explorar si el supuesto de que el grupo Ciudadanos por Michoacán se desarrollaba con base en una dinámica de una comunidad de práctica y aprendizaje, se empleó una técnica de investigación con base en los estudios de netnografía, lo que permitió observar el comportamiento de los participantes. Otro supuesto que se indagó a través de la técnica seleccionada, es la capacidad de los participantes para someter a intercambio diferentes recursos para la negociación de significados, y la capacidad de jugar diferentes roles en el grupo que generan significado, práctica, comunidad e identidad.

\subsection{REGISTRO DE LOS MENSAJES EN INTERCAMBIO}

En la primera etapa de observación y registro de las interacciones fue posible identificar el tipo de discursos que los sujetos intercambian, así como las formas y recursos que emplean para establecer sus interacciones, lo anterior permitió a su vez dar cuenta de la posibilidad de que los jóvenes se pudiesen conformar como una comunidad de práctica y reflexión, y de que estuvieran sometiendo a discusión temáticas relacionadas con la formación de ciudadanía.

En el diseño de la estrategia se generaron instrumentos permitieron indagar la relación de los textos con el contexto de los participantes del grupo, propiciando también acercamientos para la observación detallada del intercambio de mensajes que se convirtieron en interacciones. Con base en la netnografía, se registró evidencia del comportamiento cotidiano de los participantes en su propio grupo.

La observación de tipo netnográfica otorgó entre otras ventajas la sistematización de los intercambios, la observación sin intervenir directamente entre los participantes, mesurar el tiempo en el que se desarrollaron las acciones, establecer relaciones entre los participantes y rastrear las acciones de los participantes de manera histórica grupo.

Durante el trabajo de recopilación de los mensajes, se registraron todas las aportaciones y sus respuestas en el grupo Ciudadanos por Michoacán por cada día. La modalidad para generar los registros de dichas aportaciones se dio mediante el respaldó en un software para manejo de base de datos, en el que se recopilaron las aportaciones en una primera instancia como se muestra en el ejemplo de la Tabla 1.

Una vez contando con el material recopilado para la revisión de los contenidos de la comunidad virtual Ciudadanos por Michoacán, surgen las primeras inferencias que son de ayuda para la estructuración de una etapa definitiva, entre las que destacan las siguientes:

a) La participación en la producción de mensajes es mínima por parte de los integrantes del grupo, y la cantidad de mensajes está relacionada con los sucesos que acontecen en la ciudad.

b) Los temas no están encausados a una temática en específico, ni tampo- 
co están regulados por su pertinencia con la discusión en curso.

c) Se identifica una participación importante de profesionistas trabajadores del sector público del Estado de Michoacán.

d) Los profesores de la Universidad Michoacana son participantes activos en el grupo y comparten sus propias aportaciones relativas a las condiciones de la misma universidad.

e) Entre los perfiles de los usuarios que más se hacen presentes en las discusiones hay quienes motivaban las discusiones de manera cotidiana.

f) Se identifican también propuestas partidistas en abierto proselitismo, a través de difusión de las propuestas de un candidato, o bien, a manera de críticas y cuestionamientos.

g) Hay una diferenciación evidente de la participación del estudiantado universitario y normalista en la actividad de la comunidad virtual.

h) Se detecta una falta de estructura en los mensajes que permita llegar a un objetivo establecido en las discusiones.

i) Los usuarios parecen no apegarse a las reglas expuestas en la declaratoria del grupo en el que voluntariamente han aceptado participar, y su participación integra todo tipo de mensajes.

\subsection{MENSAJES RELACIONADOS CON DIMESNIONES DE CIUDADANÍA}

En el segundo momento metodológico se da una nueva recopilación de los mensajes y las interacciones dadas en el grupo Ciudadanos por Michoacán, con la intención de analizar el contenido discursivo de dichas interacciones, así como la identificación de los roles de los participantes en las discusiones.

El énfasis del segundo momento metodológico se centra en los contenidos discursivos derivados de los intercambios entre los participantes de la comunidad virtual, así, se hace la propuesta de integrar el análi- sis del discurso orientado a la estrategia de netnografía como técnica de investigación, dicha técnica se da con el propósito de hacer un reconocimiento de relaciones entre las interacciones, los perfiles de los participantes, las dimensiones de la formación de ciudadanía y las acciones de aprendizaje generadas en el ejercicio del diálogo.

Ante la dinámica de crecimiento de las discusiones y la aparición de diferentes circunstancias relacionadas con los intereses de los participantes en la comunidad, durante esta segunda etapa de registro se optó por dar una captura no cronológica, en la cual se privilegiaron las discusiones iniciales y los comentarios subordinados más allá de la temporalidad con la que estos aparecen, de esta manera la prioridad fue la configuración de discusiones que permiten dar evidencia de una verdadera interacción entre los participantes.

En la segunda etapa de registro se consiguió la captura de 70 aportaciones originales relacionadas con los criterios observables establecidos (aportaciones relacionadas con la dimensión civil, social y política de ciudadanía), lo que a su vez genera un total de 133 comentarios respuesta a las aportaciones. Como se puede observar, a diferencia de la etapa primera la cantidad de mensajes considerados y registrados fue menor, sin embargo, en este momento se trataba de aportaciones perfiladas que posteriormente fueron analizadas en búsqueda del posible proceso de aprendizaje.

Durante el proceso de registro de las aportaciones se estructuraron diferentes cédulas que se modificaron paulatinamente, hasta lograr una sola propuesta que permitió el seguimiento y correlación de los mensajes con los objetivos de la investigación. Dicho instrumento se presenta en la Tabla 2.

Con el formato de captura, mostrado en Tabla 2, se generaron 92 cédulas, una por cada día comprendido en el periodo determinado. Una vez culminado el segundo pe- 
riodo de registro se dio paso a la estructura de las conversaciones, las cuales se extraen de las cédulas de captura según la temática identificada por la aportación original y por la retroalimentación a través de los comentarios. En la Tabla 3 de este documento se muestra a la estructura de conversación reconstruida a raíz de las cédulas de registro previas.

En el registro de las interacciones orientadas a la formación de ciudadanía, y durante el proceso de reconstrucción de las conversaciones, se prestó también atención a los perfiles de los participantes activos y al papel que estos desempeñaban durante los diálogos que se establecieron en el grupo. A través de los registros de las conversaciones se analizaron los perfiles de los participantes y sus intercambios, los cuales están relacionados con los propósitos que se perseguían en su grupo, compartiendo su interés, pero también su dominio sobre conocimientos específicos correspondientes a sus necesidades en la vida cotidiana, lo que a su vez generó vínculos entre los participantes activos.

Este segundo momento metodológico de la investigación fue clave para establecer las relaciones dadas entre los integrantes de una presunta comunidad de práctica y aprendizaje a través de un grupo en Facebook, así, se generaron los análisis del contenido discursivo en las conversaciones seleccionadas, pero también se identificaron las dinámicas de participación, respuesta, réplica, negociación y construcción, de los significados, así como se sugiere en una comunidad de práctica(2).

\subsection{ASPECTOS RELACIONADOS CON ACCIONES DE APRENDIZAJE}

Dentro de la dinámica de intercambios de informaciones, recursos y símbolos por parte de los integrantes de la comunidad virtual, se le dio especial interés a las acciones que otorgaron evidencia de la existencia de acciones de aprendizaje orientadas a la formación de ciudadanía, especialmente en el caso de las tres dimensiones de ciudadanía seleccionadas, la civil, la política y la social. En relación con los observables como criterio para la construcción de las conversaciones que propiciaron acciones de aprendizaje, se tomó la referencia de una serie de acciones previamente desarrollados dentro del Basic Support for Cooperative Learning (BSCL), que en su momento funcionó para identificar los modelos pedagógicos para la construcción del conocimiento colaborativo, y que a su vez tiene base en la teoría de cognición grupal de Stahl (en Cros Salavat, 2008:108).

Se hace referencia a la teoría de la cognición grupal de Sthal, debido a que precisamente bajo esta perspectiva se propone que el conocimiento es el resultado de las interacciones individuales en el nivel grupal, y que los grupos construyen un conocimiento que no pueden gestarse en ninguna mente individual, ya que el conocimiento se logra a través del discurso grupal y se mantiene a través de artefactos físicos y simbólicos, como el caso de las mediaciones tecnológicas.

Para Stahl, "el conocimiento es el resultado de los procesos colaborativos donde se negocian diferentes perspectivas interpretativas. El aprendizaje colaborativo es un proceso de construcción de significados que consiste en crear una red de significados en un grupo" (Stahl en Cros, 2008: 85). Estas afirmaciones toman valor para la presente investigación debido a que bajo esta lógica, el propio Stahl afirma que en los grupos pequeños se genera conocimiento, no como la suma de las aportaciones individuales, sino como el resultado de las interacciones en el grupo.

La noción de cognición grupal se concibe como un aspecto metodológico más que permite tomar al grupo como unidad de análisis. De esta manera el conocimiento producido en el grupo es apreciable a través del mismo discurso empleado en las interacciones de los integrantes como parte de un todo. Por lo anterior, el conocimiento logrado en el grupo rebasa al que se pudie- 
se propiciar por los individuos como ejercicio individual.

En el caso del grupo Ciudadanos por Michoacán, que es objeto de este estudio, las interacciones seleccionadas para observar fueron aquellas interacciones con contenidos discursivos orientados a la formación de ciudadanía, y que daban evidencia de algunas de las acciones relacionadas con el proceso de aprendizaje tomado como base. Dichas acciones orientadas al proceso de aprendizaje, y su propuesta de análisis, se presentan en la Tabla 4.

Una vez ubicados aquellos mensajes que sí propiciaban relación con los objetivos de la investigación, a todos los mensajes seleccionados se les señalaron los elementos léxico-semánticos(3) para su posterior relación con las garantías individuales y las dimensiones de ciudadanía propuestas.

Específicamente en este análisis se le prestó atención a los vocablos que hicieron referencia a una preocupación relacionada con las garantías individuales. Lo anterior debido a que el léxico tiene una función cohesiva sustancial en la estructuración del contenido del texto, además, a través del lenguaje se da la negociación de los significados, incluyendo las relaciones sociales y el uso de los espacios como factores de negociación, lo que a decir de Wenger supone un proceso activo y la capacidad de influir entre los participantes en la comunidad de práctica (Wenger, 2001: 78).

Como análisis de tipo semántico se precisó la interpretación de las palabras empleadas en función de su significado, ya que es lo que le da el sentido a las frases y por tanto a los mensajes, en el mismo sentido el contenido semántico de las frases se encuentra determinado por el contexto y el momento de los usuarios. Este tipo de señalamientos en el discurso permitió reconocer la forma con la que los miembros del grupo estructuraban sus ideas.
Así, se comprende que la práctica es ese hacer dentro del contexto histórico y social, en el que se le otorga estructura y significado a eso que se hace, en este caso, a las interacciones relacionadas con la formación de ciudadanía a través del entorno virtual Facebook.

\section{RESULTADOS}

Una vez desarrolladas las etapas de registro se dio paso al análisis de los mensajes, articulados ya como conversación, en donde se observaron los siguientes resultados:

a)Las interacciones comunicativas generadas entre los sujetos de estudio durante los momentos de la observación para esta investigación, estuvieron definidos por esos procesos de socialización y la construcción de diálogos negociados por los mismos miembros de la comunidad virtual en la que participan.

b)En el caso de la comunidad Ciudadanos por Michoacán, la socialización fue apreciada como el inicio de una serie de negociaciones en las que los significados en torno a un interés común se expusieron y se cuestionaron a la vez.

c)La dinámica de exposición, cuestionamiento, y negociación del significado fue característica del periodo en el que transcurrieron las campañas políticas de años 2011 y 2012, lo anterior debido a que la participación en la comunidad virtual incrementó mediante el intercambio constante de mensajes de diversa índole, mensajes entre los que destacan los relacionados con los comicios electorales principalmente, pero también surgieron algunos mensajes relacionados con el interés de los jóvenes por la formación de ciudadanía.

Dichos hallazgos, se construyeron con base en una serie de matices que pasan por los aspectos de tipo lingüístico, semántico y/o interaccional. En algunos casos se observa en las participaciones que la redacción y la 
claridad en la exposición permiten la identificación de una idea, sin embargo, en otros ejemplos la información dispuesta para la participación parece ser una palabra, o conjunto de palabras, sin significado claro y sin una estructura efectiva en el contexto del grupo quedando como una simple expresión.

En el caso de las expresiones cortas de los participantes, éstas parecen no tener importancia para su análisis como mensaje individual, incluso en la mayoría de las ocasiones no se les logra relacionar con ninguna dimensión de ciudadanía ni con alguna garantía en la constitución. Sin embargo, las mismas expresiones cortas generaron un sentido y portaron un significado muy diferente al momento de integrarlas como componentes en las conversaciones.

Una característica que se observa en el trabajo de análisis con los mensajes de manera individual, es la falta de homogeneidad en el uso del lenguaje entre los participantes. En cuanto a su sentido morfológico y semántico se aprecian algunos mensajes escritos en forma de denuncia, otros a manera petición y otros en un formato de defensa, pero todos con diferencias en la manera de construir la oración que someten como participación.

Otro aspecto a considerar en el análisis es el tiempo que los usuarios emplean para generar sus aportaciones, en su mayoría los usuarios del grupo parecen generar sus aportaciones de manera espontánea, con gran cantidad de descuidos en la construcción de la aportación, y en menor medida, se identifican también aquellos usuarios que generan mensajes más cuidados, quienes incorporan recursos y referencias de refuerzo para sus argumentos.

En este orden de ideas se prestó atención al aspecto relacionado con el tiempo de participación, debido a que los usuarios que verdaderamente generan aportaciones con fines de contribuir a las discusiones de sus pares, dedican mayor tiempo para construir ideas más largas, claras y, en la mayoría de los casos, con las debidas referencias que sustentan sus afirmaciones. En este sentido, se detectaron también algunos perfiles que ostentan cierto liderazgo en las participaciones del grupo, identificados públicamente como estudiantes de la Universidad Michoacana de San Nicolás de Hidalgo.

Varias de las aportaciones coincidieron con diferentes garantías individuales y con más de una dimensión de ciudadanía, lo que dio muestra de la coincidencia de las preocupaciones por parte de los integrantes del grupo Ciudadanos por Michoacán y las condiciones apreciadas en el contexto del estado. Durante la investigación se prestó atención a las pocas aportaciones que construyeron una idea compleja, que se encontraba relacionada con las dimensiones de ciudadanía y que fue sometida a discusión entre los usuarios del grupo.

En lo relacionado con los jóvenes universitarios se pudo apreciar cómo contribuyeron con sus mensajes, más a través de las respuestas generadas en una discusión que a través de aportaciones originales. Llama la atención una diferencia muy evidente entre sus mensajes incorporados a las discusiones de otros adultos y los mensajes que ellos emiten y dirigen hacia sus pares.

En el caso de los mensajes de los jóvenes dirigidos a otros jóvenes se observó una mayor estructura y una mayor intención por propiciar el diálogo, en algunos incluso se dio un manejo de referencias, recursos y hasta posicionamientos sobre el tema. Con lo anterior, una de las primeas inferencias es la relativa a la dinámica establecida entre los jóvenes con los otros jóvenes, con los que incluso conviven en otros espacios de la vida presencial, en contraste con la dinámica de participación efímera e intermitente en las discusiones de otros usuarios adultos. 


\section{DISCUSIÓN}

En las conversaciones analizadas dentro del grupo Ciudadanos por Michoacán en Facebook, la condición de los estudiantes nicolaitas con mayor participación, en un carácter casi protagónico, llamó la atención debido a sus marcadas diferencias con otros de su misma edad también integrantes de la comunidad virtual.

Debido a las condiciones de aportación en los mensajes y al establecimiento de la dinámica en las discusiones por parte de los jóvenes protagonistas en las discusiones presentadas, resultó interesante indagar sobre las relaciones de dichos jóvenes universitarios en otros ámbitos de su vida, así, toda información recopilada fue obtenida a través del mismo entorno Facebook y en su propia publicación de perfil público.

Entre las condiciones encontradas, relacionadas con su perfil público en el mismo Facebook, se tiene que son estudiantes que comparten tiempo entre sí en otras actividades diferentes a las académicas, como las de participación activista a favor de la ecología, de los animales, en contra de la violencia, y otras. Dos de los jóvenes participantes son miembros del H. Consejo Técnico de su dependencia universitaria, y uno es miembro del H. Consejo Universitario, máximo órgano de decisión en la Universidad Michoacana de San Nicolás de Hidalgo. Todos se declaran de perfil apartidista y corresponden a las áreas de estudio de derecho, administración, medicina y psicología.

En las conversaciones de la comunidad virtual Ciudadanos por Michoacán, en las que solamente participan dichos jóvenes universitarios, se pudo a apreciar una ligera transformación de los contenidos deliberados, especialmente por aquellos que inician la disertación en la exposición de su preocupación o necesidad. No es el caso de otras conversaciones en las que los actores son desconocidos para ellos en el mundo presencial, en tales conversaciones, se limitan a la participación incidental sin dar segui- miento a la negociación de las perspectivas, simplemente las abandonan o cambian de asunto a tratar en la comunidad virtual. Esta condición, de conocimiento previo y de interacción en el mundo presencial por parte de los citados estudiantes, permitió desarrollar una inferencia en sentido de que las mediaciones desarrolladas entre los jóvenes puedan prosperar, o no, dependiendo de sus relaciones establecidas en otros escenarios del mundo presencial.

Con el corpus de mensajes recopilado se reconstruyeron 6 líneas conversacionales relacionadas con la formación de ciudadanía, sin embargo, se sometieron a análisis en esta investigación solamente 5(4) debido a que una secuencia no se pudo completar en una línea conversacional, lo anterior como resultado de la desaparición de varias de las participaciones por parte de los usuarios.

Otra de las inferencias parciales derivadas de esta etapa de análisis, fue que la dimensión de ciudadanía más presente en estas conversaciones fue la dimensión civil, seguida de la dimensión política, y finalmente la dimensión social.

Por otro lado, la mayoría de las conversaciones presentaron acciones del proceso de aprendizaje en sus etapas básicas, como las de articulación de la idea, de contribución pública y de contribución de otros participantes del grupo, y pocas fueron aquellas que trascendieron hasta las acciones de argumentación, clarificación de los significados, negociación de perspectivas y símbolos empleados. Ninguna conversación otorgó evidencia clara de llegar a la última acción del proceso de construcción del conocimiento colaborativo, como se muestra en la misma Tabla 4 del presente documento.

Se reconoce también, como parte de la discusión vigente, la dificultad que tuvo la presente investigación para evidenciar la posible formación entre pares dentro de una 
comunidad de práctica con las características señaladas, la cual se encuentra conformada por jóvenes con diferentes niveles de conocimiento sobre las dimensiones civil, política y social de la formación de ciudadanía.

Desde la mencionada complejidad, en esta investigación se aportan también las siguientes reflexiones sobre la tecnología, educación para la ciudadanía y los jóvenes nicolaitas, desde las cuales se pueden desprender nuevas indagatorias de investigación relacionadas con la discusión sobre el tema.

Debido a que los jóvenes universitarios son usuarios con altos niveles de conectividad a través de diferentes dispositivos, se identificó como una posibilidad de estudio la observación del alumnado con énfasis en la distinción de los diferentes dispositivos y los diferentes entornos virtuales de los que son usuarios, así, podría indagarse sobre la discriminación de los entornos a disposición en función de sus objetivos de uso. Esta observación coincide con lo reportado por Crovi et. al. (2013: 45) respecto a otros jóvenes universitarios del país

De igual manera cabe la oportunidad de cuestionamientos como: ¿cuáles son los criterios que influyen en los jóvenes para el uso de una red social u otras? En su caso ¿qué determina su migración entre éstas? $\mathrm{O}$ bien ¿Hay relación entre las afiliaciones políticas, la condición económica social, las actividades del entorno presencial y las condiciones de participación activista en las redes sociales en línea?

Los pocos jóvenes que sí propiciaron actividades relacionadas con el proceso de aprendizaje, durante su participación en el entorno virtual Facebook, se mostraron en un rol de evidente liderazgo dentro y fuera del entorno. Con este antecedente surge la inquietud de problematizar las posibilidades de un ejercicio de educación estructurada entre pares con una temática específica, en el cual se pudiera disponer de variables controladas con la participación de jóvenes siendo educados por otros jóvenes, dicha suposición podría aportar evidencias más contundentes sobre la posibilidad de actos educativos y la adquisición de conocimientos a través de entornos como Facebook.

Por otro lado, se pudo apreciar que los jóvenes suelen utilizar el entorno Facebook como extensión de sus relaciones presenciales, independientemente de que el entorno virtual ofrece la posibilidad de conexión con cualquier usuario de la misma red, en la práctica los usuarios (así en los usuarios nicolaitas observados) parecen fomentar extensiones, de tiempo, distancia, contenido y hasta de capacidades de diálogo en relaciones establecidas previamente.

En continuidad con lo anterior, una de las posibles indagatorias que se desprenden de este hecho es: ¿qué tipo de evidencia permitiría sugerir que los jóvenes nicolaitas pueden crear asociaciones colaborativas con otros jóvenes, a quienes no conocen en el escenario presencial, pero con los que sí comparten las mismas preocupaciones e iniciativas?

Como se ha visto, los jóvenes michoacanos que participan constantemente en las redes sociales en línea someten a intercambio muchos temas que están directamente relacionados con los acontecimientos de su realidad, a pesar de esto, no existe evidencia que permita comprobar la relación directa entre la participación de tipo política de los jóvenes en los entornos virtuales, y la participación tangible en los escenarios presenciales.

Como se decía, una vez detectados aquellos jóvenes que sí establecen conversaciones más complejas con el intercambio de símbolos y posturas relacionadas con la formación de ciudadanía, queda la inquietud por conocer ¿cuáles son las condiciones de sus biografías que les permiten someter a negociación ese tipo de intereses que marcan una diferencia evidente con el resto de sus pares? 


\section{CONCLUSIONES}

El presente trabajo de investigación ha tratado la manera en la que los jóvenes universitarios de Michoacán generan participaciones a través de las redes sociales, con las que propician interacciones que a su vez construyen conversaciones más complejas, y en las cuales dan evidencia de la existencia de ciertas acciones relacionadas con el proceso de enseñanza y aprendizaje entre pares.

Específicamente, se observó el aspecto educativo relacionado con la acepción y formación de las dimensiones civil, social y política de la ciudadanía, esto en una comunidad ciudadana constituida a través de la red social en línea Facebook.

La investigación partió de una indagatoria central que cuestiona la realidad y las posibilidades de los jóvenes universitarios de Michoacán reconocidos como usuarios de la tecnología, y que se hacen presentes a través de las redes sociales en línea, dentro de las cuales dan muestra de las condiciones de intercambio que establecen con sus pares y con otros usuarios. Así, como parte medular de estas conclusiones, se ponen a disposición las siguientes afirmaciones como producto del trabajo en sus diferentes etapas.

Los jóvenes universitarios observados en el grupo Ciudadanos por Michoacán de Facebook dan evidencia de socializar sus preocupaciones a través de sus participaciones en internet, no sólo en los espacios reconocidos como personales (como en el muro de Facebook) sino en foros de carácter público como es el caso de del grupo para la ciudadanía en el que figuran otros participantes del mundo adulto que se expresan sobre los asuntos públicos de la sociedad.

Se identifica una clara diferenciación entre el carácter de los mensajes publicados por los jóvenes en espacios personales y los expuestos en el foro público, estos últimos se describen con más cuidados en su forma retórica de exposición.
Los jóvenes muestran la capacidad de confrontar las ideas y argumentaciones provenientes de otros usuarios adultos. Este dato llama la atención en función de su poca probabilidad de ocurrencia en el espacio presencial en el que la confrontación no suele darse de manera abierta. Con lo anterior, se refuerza la noción del espacio virtual como un complemento y no como un entorno independiente a los acontecimientos del mundo presencial.

Los estudiantes miembros del grupo en observación muestran la habilidad para integrar recursos informativos en distintos formatos, recursos que fortalecen sus perspectivas sobre tema y que permiten generar evidencias para incluir a sus pares a la discusión del asunto.

Los nicolaitas objeto de la observación propician acciones del proceso de aprendizaje. En algunos casos los jóvenes incorporan para su discusión recursos reconocidos como oficiales, como la Constitución Política o los comunicados del Poder Ejecutivo y Legislativo por ejemplo, pero en muchos otros casos se observa que incorporan recursos de tipo alternativo que son producidos por los mismos jóvenes con elementos más atractivos para sus pares, es el caso de los blogs, páginas en Facebook, videos, animaciones y memes.

En las discusiones clave del análisis, aquellas en las que participaron jóvenes universitarios de Michoacán en temáticas relacionadas con las dimensiones civil, social y política de ciudadanía, se encuentra evidencia de que los jóvenes se muestran altamente interesados en las condiciones relacionadas con sus garantías individuales.

En el discurso que los jóvenes emplean para referirse a las prerrogativas del ciudadano y sus garantías individuales, no se emplea un léxico de formalidad, por el contrario los jóvenes suelen entablar las discusiones relacionadas utilizando sus propios referentes 
en los que se da la construcción de nuevos símbolos.

En las conversaciones analizadas se identificaron acciones correspondientes al proceso de aprendizaje colaborativo tomado como base para la investigación, en las cuales, las acciones del proceso de aprendizaje más recurrentes son las de articulación de la idea, contribución pública, contribución de otras personas del grupo y argumentación. Solamente en un par de las conversaciones analizadas los jóvenes nicolaitas dan evidencia de alcanzar las acciones de clarificación de significados, símbolos empleados y negociación de perspectivas.

Con todo lo expuesto en este reporte del trabajo de investigación, se tiene la certeza de que dichos jóvenes desarrollan y propician acciones relacionadas con el aprendizaje de ellos mismos y sus pares en relación a la formación de ciudadanía, sin embargo, no hay evidencia de que exista nuevo aprendizaje a partir de las interacciones observadas y analizadas dentro del grupo Ciudadanos por Michoacán, por lo que se sugiere dar continuidad a las indagatorias, desde otras perspectivas que pudieran ser complementarias al presente estudio.

\section{REFERENCIAS BIBLIOGRÁFICAS}

- Cabrera G. (2014). Apropiación del software social en Internet de los alumnos nicolaitas del área de la salud. (Tesis de Licenciatura), Universidad Michoacana de San Nicolás de Hidalgo. Morelia, Michoacán, México.

- Castells, M. (2012). Redes de indignación y esperanza: Los movimientos sociales en la era de internet. Madrid: Alianza Editorial.

- Cros B. (2008). Aprendiajes, conexiones y artefactos. La producción colaborativa del conocimiento. Barcelona, España: Gedisa.

- Crovi, D., Garay, L. M., López, R., y PortiIlo, M. (2013). Jóvenes y apropiación tecnológica: La vida como hipertexto. Méxi- co: Sitesa/UNAM, Facultad de Ciencias Políticas y Sociales.

- Cruz L. (2013). Apropiación del Software Social Facebook por parte de los alumnos de la Universidad Michoacana. (Tesis de Licenciatura), Universidad Michoacana de San Nicolás de Hidalgo. Morelia, Michoacán, México.

- Fernández, A. (2010). Formación de ciudadanía e identidad. Voces de la sociedad civil. México DF: Universidad Pedagógica Nacional.

- Habermas, J. (1999). Teoría de la acción comunicativa, I: Racionalidad de la acción y racionalización social. Buenos Aires: Taurus Humanidades.

- Padilla M. (2013). El sentido de las prácticas políticas de los jóvenes en Internet. Análisis de la EJIPP 2012. En Cornejo y Guadarrama (Coord.), Culturas en comunicación: Entre la vocación intercultural y las tecnologías de información (pp. 127-152). México: Editorial Tintable.

- Robinson, W. (2007). Una teoría sobre el capitalismo global. Producción, clase y Estado en un mundo transnacional. Bogotá, Colombia: Ediciones los de abajo.

- Sarramona, J., Vázquez, G., \& Colom, C. A. J. (1998). Educación no formal. BarceIona: Ariel.

- Turpo, O. (2011). La netnografía: un método de investigación en Internet. En Revista Iberoamericana de Educación, Universidad de Salamanca, España, No. 47, Octubre, 1-10. Recuperado de: http:// www.rieoei.org/deloslectores/2486Gebera.pdf

- Wenger, E. (2001). Comunidades de práctica: Aprendizaje significado e identidad (G. Sánchez, Trad.). Barcelona, España: Paidós Ibérica. 
Tabla 1. Ejemplo de la primera cédula del instrumento de registro en la primera etapa

\begin{tabular}{|l|l|l|l|}
\hline SUJETO & APORTACIÓN & TEMÁTICA & FECHA \\
\hline & & & \\
\hline
\end{tabular}

Tabla 2. Ejemplo de cédula de registro utilizada en el periodo final de captura

\begin{tabular}{|l|l|l|l|l|l|l|}
\hline Nomenclatura & Sujeto & Papel & Aportación & Tema & Respuesta a: & Fecha \\
\hline & & & & & & \\
\hline
\end{tabular}

Tabla 3. Ejemplo de conversación reconstruida con base en la cédula de análisis (5)

\begin{tabular}{|l|}
\hline CONVERSACIÓN 160512 \\
\hline Fernando $\{$ APORTACIÓN ORIGINAL\} \\
\hline 1310_160512 Juan Carlos \{COMENTARIO RESPUESTA \\
\hline 1349_160512 Chilo $\{$ COMENTARIO RESPUESTA $\}$ \\
\hline 1400_160512 Juan $\{$ COMENTARIO RESPUESTA $\}$ \\
\hline 1403_160512 Chilo $\{$ COMENTARIO RESPUESTA $\}$ \\
\hline 1958_180512 \{COMENTARIO RESPUESTA $\}$ \\
\hline
\end{tabular}

Tabla 4. Descripción de las acciones, con base en el Basic Supportfor Cooperative Learning, como parte del proceso de aprendizaje buscado en la comunidad virtual (6)

\begin{tabular}{|l|l|}
\hline Acciones observadas en las interacciones & $\begin{array}{l}\text { Descripción en el propósito del análisis } \\
\text { (Adaptados desde la postura de Cros, 2008) }\end{array}$ \\
\hline Articulación de la idea & $\begin{array}{l}\text { Las intenciones de contribución para el } \\
\text { grupo que tiene cada usuario, su construc- } \\
\text { ción creativa es un referente del área del } \\
\text { conocimiento que domina y de su expe- } \\
\text { riencia en las relaciones con otros usuarios } \\
\text { y con el entorno, real y virtual. }\end{array}$ \\
\hline Contribución pública & $\begin{array}{l}\text { La manera en la que el usuario materializa } \\
\text { la idea a compartir con el grupo. En esta } \\
\text { acción se refleja el formato en que se alo- } \\
\text { ja el contenido, texto, vínculo, audio, video } \\
\text { etc. Así como la estructuración del discurso } \\
\text { que se emplea, el que se aprecian posicio- } \\
\text { namientos del usuario respecto a la con- } \\
\text { cepción de los otros. }\end{array}$ \\
\hline
\end{tabular}




\begin{tabular}{|c|c|}
\hline Contribución de otras personas del grupo & $\begin{array}{l}\text { Se trata de la reacción, o no, de los otros } \\
\text { usuarios con respecto a la contribución ori- } \\
\text { ginal, marcando una tendencia de refuerzo, } \\
\text { cuestionamiento u oposición. Fomentando } \\
\text { una dinámica para la continuidad temática } \\
\text { por el mismo autor u otros usuarios que pu- } \\
\text { diesen integrar. }\end{array}$ \\
\hline Argumentación & $\begin{array}{l}\text { Es el momento, de mayor complejidad en } \\
\text { el acto individual, en el que el participante } \\
\text { se vale de demostraciones explícitas que } \\
\text { permiten dar validez a una verdad en la dis- } \\
\text { cusión. Especialmente se considera como } \\
\text { aquella afirmación sostenida con razones, y } \\
\text { de acuerdo con Habermas (1999) es resul- } \\
\text { tado del proceso, del procedimiento y de la } \\
\text { propia producción del hablante. }\end{array}$ \\
\hline Clarificación de significados & $\begin{array}{l}\text { Los usuarios participantes en la discusión } \\
\text { toman postura y se identifican con una } \\
\text { apreciación sobre el objeto de la discusión, } \\
\text { aceptando abiertamente estar en concor- } \\
\text { dancia con una idea, o bien, diferir sobre } \\
\text { algunas contribuciones. }\end{array}$ \\
\hline Símbolos empleados & $\begin{array}{l}\text { Se toma en cuenta los signos socialmen- } \\
\text { te convenidos que se ponen a negociación } \\
\text { entre los participantes, otorgándoles un } \\
\text { peso y una trascendencia particular depen- } \\
\text { diente del clima de la discusión. Así los sím- } \\
\text { bolos lingüísticos como los iconográficos. }\end{array}$ \\
\hline Negociación de perspectivas & $\begin{array}{l}\text { Se da cuando alguno de los participantes } \\
\text { muestra interés por comprender los demás } \\
\text { argumentos, que en un ejercicio más allá } \\
\text { de ceder, se propone reconstruir un sig- } \\
\text { nificado que permita la integración de las } \\
\text { perspectivas de otros usuarios, y no sola- } \\
\text { mente la propia. }\end{array}$ \\
\hline Conocimiento colaborativo & $\begin{array}{l}\text { Se puede observar en el momento que } \\
\text { el nuevo significado se ha estructurado } \\
\text { y queda listo para someterlo a una nueva } \\
\text { deconstrucción, en donde habrán de parti- } \\
\text { cipar nuevos actores con perspectivas dis- } \\
\text { tintas en un momento determinado, some- } \\
\text { tiéndolo a nuevas negociaciones. }\end{array}$ \\
\hline
\end{tabular}




\section{NOTAS:}

(1) Nicolaitas, es la manera coloquial con la que se suele nombrar a los estudiantes que pertenecen a la Universidad Michoacana de san Nicolás de Hidalgo en la ciudad de Morelia Michoacán.

(2) Para identificar la categoría de comunidad de práctica y aprendizaje incorporada por esta investigación se recomienda la revisión del subtema

(3)Por elementos léxicos en el discurso se entienden aquellos vocablos incorporados al texto, que son parte del lecto de los hablantes

(4)Se presume, como posibilidad, que al calor del intercambio de varios mensajes relacionados con los comicios electorales varios de los participantes incurrieron en ataques y descalificaciones directas hacia otros, por lo que los administradores del grupo pudieron retirar dichos mensajes de los registros, al no contar con esta evidencia se decidió en la investigación presentar la información solamente como parte de la estadística.

(5) En este cuadro se aprecia la manera en la que se construyeron las conversaciones que se nutren de las diferentes aportaciones detectadas en las cédulas de registro, se puede identificar cómo cada uno de los mensajes se genera en un momento distinto pero dada la relación jerárquica que se le asigna y la nomenclatura en función de su aparición permite reconstruir una secuencia de mensajes.

(6) Las acciones revisadas en el Cuadro 4, pueden presentarse todas o solamente algunas dependiendo de la dinámica del proceso de construcción, igualmente el orden de las etapas no necesariamente es cronológico, incluso en algunos casos puede estar cargada con gran cantidad de participaciones todas en la misma acción

\section{CURRÍCULUM VITAE:}

Dr. Alberto Farías Ochoa,

Profesor Investigador Asociado " $\mathrm{C}$ ", en la Facultad de Letras de la Universidad Michoacana de San Nicolás de Hidalgo. Miembro de la Asociación Mexicana de Investigadores de la Comunicación. Miembro de la Asociación Latinoamericana de Estudios del Discurso. Integrante del Núcleo Académico Básico del Posgrado en Acceso a la Información de la UMSNH. Integrante del Núcleo Académico Básico del Posgrado en Estudios del Discurso de la UMSNH. Trabaja las líneas de investigación de: comunicación intercultural; de comunicación y tecnologías; y de comunicación educación. 